\title{
Photonics-based ultra-low phase noise X-band microwave generation
}

L. Karlen, N. Torcheboeuf, S. Kundermann, T. Herr, E. Obrzud, et al.

L. Karlen, N. Torcheboeuf, S. Kundermann, T. Herr, E. Obrzud, V. Brasch, F. Schaub, H. Meier, A. Stroganov, M. Geiselmann, S. Lecomte, "Photonicsbased ultra-low phase noise X-band microwave generation," Proc. SPIE 11852, International Conference on Space Optics — ICSO 2020, 1185246 (11 June 2021); doi: 10.1117/12.2599641

SPIE Event: International Conference on Space Optics - ICSO 2021, 2021, Online Only 


\section{International Conference on Space Optics-ICSO 2020}

Virtual Conference

30 March-2 April 2021

Edited by Bruno Cugny, Zoran Sodnik, and Nikos Karafolas
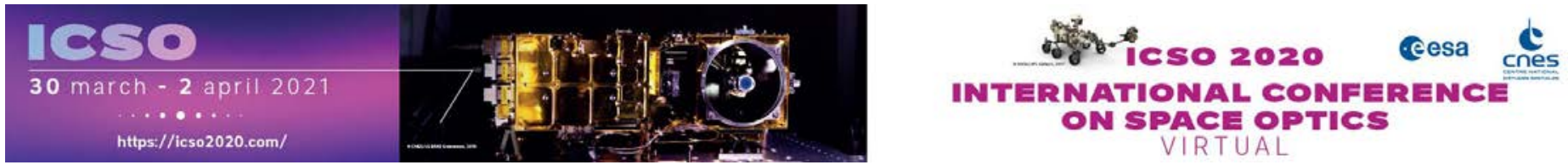

\section{Photonics-based ultra-low phase noise $X$-band microwave generation}

\section{Cesa isoporecestings denes}




\title{
Photonics-based ultra-low phase noise $\mathrm{X}$-band microwave generation
}

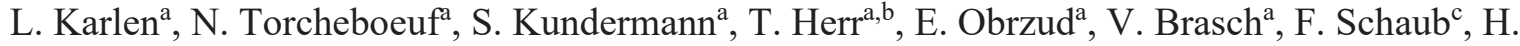 \\ Meier $^{c}$, A. Stroganov ${ }^{\text {d, e }}$, M. Geiselmann ${ }^{\text {d }}$ S. Lecomte*a \\ ${ }^{a}$ Centre Suisse d'Electronique et de Microtechnique (CSEM), Jaquet-Droz 1, 2000 Neuchâtel, \\ Switzerland \\ ${ }^{\mathrm{b}}$ Current address : Center for Free-Electron Laser Science (CFEL)/German Electron Synchrotron \\ (DESY), Notkestr. 85, 22609 Hamburg, Germany \\ ${ }^{c}$ Albis Optoelectronics AG, Moosstrasse 2a, 8803 Rueschlikon, Switzerland \\ d LIGENTEC, EPFL Innovation Park Bâtiment L, Chemin de la Dent d'Oche 1B, 1024 Ecublens, \\ Switzerland \\ e ITMO University, Kronverksky pr. 49, 197101, St-Petersburg, Russia
}

*steve.lecomte@csem.ch; www.csem.ch

\begin{abstract}
Mode-locked lasers, and in particular solid-state femtosecond lasers, are oscillators with a unique physics capable to exhibit extremely low-phase noise of the emitted pulse train. Here we report ultra-low phase noise microwave generation with a self-referenced, fully-stabilized mode-locked femtosecond laser. The system involves a $395-\mathrm{MHz}$ repetition rate $1560 \mathrm{~nm}$ laser which is self-referenced and whose repetition rate is locked to a cavity-stabilized continuous-wave laser. The selfreferencing is achieved with a f-3f nonlinear interferometer realized in a silicon nitride highly-nonlinear waveguide. Prior to optical-to-electrical conversion for X-band microwave generation, the 395-MHz repetition rate of the mode-locked laser pulse train is multiplied in a fiber interleaver to $3.16 \mathrm{GHz}$. A high-power handling photodiode converts the optical pulse train to an electrical frequency comb with $3.16 \mathrm{GHz}$ frequency spacing. Finally, the $9.5-\mathrm{GHz}$ harmonics is bandpassfiltered and phase noise measurements have shown a record-low phase noise floor of $-175 \mathrm{dBc} / \mathrm{Hz}$ at $1-\mathrm{MHz}$ offset frequency.
\end{abstract}

Keywords: microwave, mode-locked laser, phase noise, photodiode, silicon nitride waveguide

\section{INTRODUCTION}

Microwave oscillators are ubiquitous in many satellites used for satcom, Earth observation and science instruments as they are necessary for on-board clock generation, phase-array antennas for beam forming, radars, etc. The phase noise of these oscillators is in many cases critical and photonics offers new capabilities to generate ultra-low phase noise microwave signals at $\mathrm{GHz}$ or tens of $\mathrm{GHz}$ carrier frequencies. The typical scheme involves a photonics oscillator and a photodiode. The optical-to-electrical conversion performed by the photodiode can be located next to the photonics oscillator or can be "on-site" by transporting the light via low-loss light-weight optical fibers. For instance, the photodetector can be located close to a microwave antenna while the photonics oscillator can be kept at a central location such that the light (and hence the microwave clock signal) is distributed via optical fibers. This last feature is opening the way to robust, low propagation loss and high-performance clock signal distribution onboard a satellite. Many different photonic oscillators and photodiodes types have been demonstrated for such applications: for instance opto-electronic oscillators [1], beating of two cw lasers [2], and mode-locked lasers [3]. Here we select the two respective technologies to achieve the best phase noise performances, i.e. a mode-locked laser based on solid-state technology and a high-power handling photodiode. Indeed, thanks to its intrinsic physics (low quantum noise), solid-state mode-locked lasers are known to provide the best phase noise performances of all kinds of oscillators (like quartz, SAW, YIG, sapphire, optical microresonator, etc.) [4] [5]. The photodetection is achieved with a high-speed high-power handling photodiode from Albis Optoelectronics (see related contribution to this conference entitled "High Power Photoreceiver for Large Dynamic Range - High Frequency Photonic 
RF Links") which allows avoiding saturation of the photodiode at high photocurrent and hence reducing the contribution of the shot noise to the phase noise while simultaneously generating microwave power on the order of $0 \mathrm{dBm}$. In addition and in the prospect of minimizing the size, weight and power (SWaP) of the system, the self-referencing of the modelocked laser is implemented with a silicon nitride waveguide which allows the $\mathrm{f}-3 \mathrm{f}$ nonlinear interferometer in one compact device.

These proceedings are organized as follows: first the system is described then the different sub-systems like the modelocked laser, the erbium-doped fiber amplifier, the pulse interleaver, the silicon nitride waveguide and the high-power handling photodiode are introduced. Finally, the obtained phase noise results are presented followed by a conclusion.

\section{SYSTEM DESIGN}

At the core of the proposed system, is a femtosecond mode-locked laser (MLL) developed by CSEM and based on lownoise diode-pumped solid-state technology mode-locked with a semiconductor saturable absorber mirror (SESAM). The laser operates at a wavelength of $1.56 \mu \mathrm{m}$ (optical signal 1 in Figure 2-1). It is amplified in a parabolic erbium-doped fiber amplifier (EDFA) to provide sufficient pulse peak power for thef- $3 f$ interferometer on one hand and for the RF generation on the other hand. Upstream the amplifier, a small amount of light is sampled for the stabilization of the laser repetition rate $f_{\text {rep. }}$.

The EDFA output is split into two parts: one part serves as an input signal for the monolithically integrated photonicsintegrated circuit (PIC) based f-3f interferometer for the carrier-envelope offset frequency $f_{0}$ detection (optical signal 2) and a second part is used for pulse interleaving (optical signal 4) and RF generation (optical signal 5) in the high-power handling photodiode (HP-PD). The fiber between the fiber splitter and the input of the PIC waveguide from Ligentec serves to compress the optical pulses and its length is optimized to get the shortest optical pulses possible. The PIC generates more than one octave spanning supercontinuum and a third harmonic in the same device thus allowing to generate the optical signal (6) necessary for the $f_{0}$ beat note detection in a dedicated, high-sensitivity, low-power photodiode.

The second output of the fiber splitter (optical signal 4) is fed into a three-stage pulse interleaver, which is made of polarization-maintaining (PM) fiber with precise delay adjustment. At the output of the interleaver (optical signal 5) the optical pulses are temporally stretched due to the dispersion of the long fiber stretch. In order to recompress the pulses impinging on the Albis HP-PD, a piece of dispersion compensation fiber (DCF) is inserted (optical signal 6). At the HPPD output, an RF signal carrying the fundamental of the $3.16 \mathrm{GHz}$ and its harmonics as well as the spurious harmonics of the laser $395-\mathrm{MHz}$ repetition rate is available. The third harmonic of the $3.16 \mathrm{GHz}$ (i.e. $9.5 \mathrm{GHz}$ ) is analyzed regarding its phase noise.

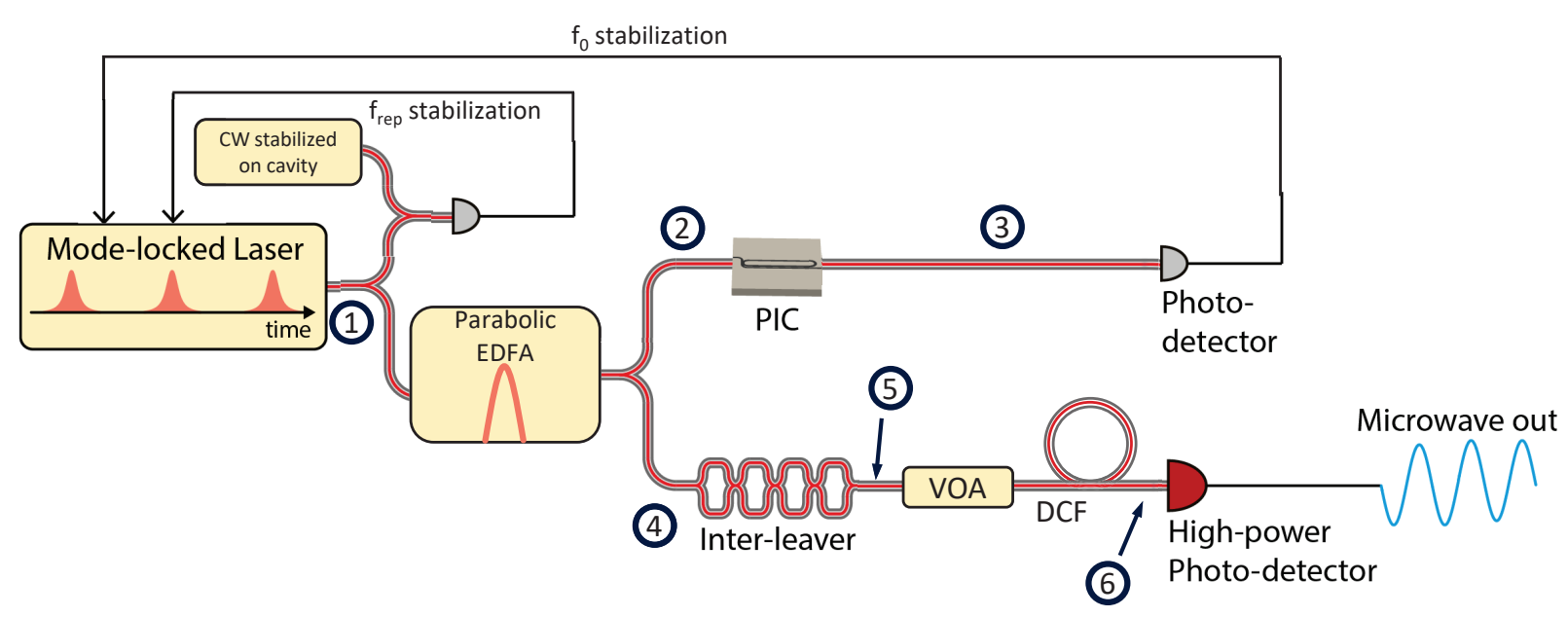

Figure 2-1 : Schematic overview of the realized photonics-based microwave oscillator. CW: continuous wave, VOA: variable optical attenuator, EDFA: erbium-doped fiber amplifier, PIC: photonic-integrated circuit, DCF: dispersion compensating fiber. 


\section{SUB-SYSTEMS DESIGNS}

\section{Mode-locked laser}

The mode-locked laser was fully developed at CSEM. A photo of a laser from the same series is shown in Figure 3-1. The laser is in a ruggedized package. All optical and electrical interfaces are situated on the same face of the housing. The laser optical output is delivered by a PM fiber pigtail.

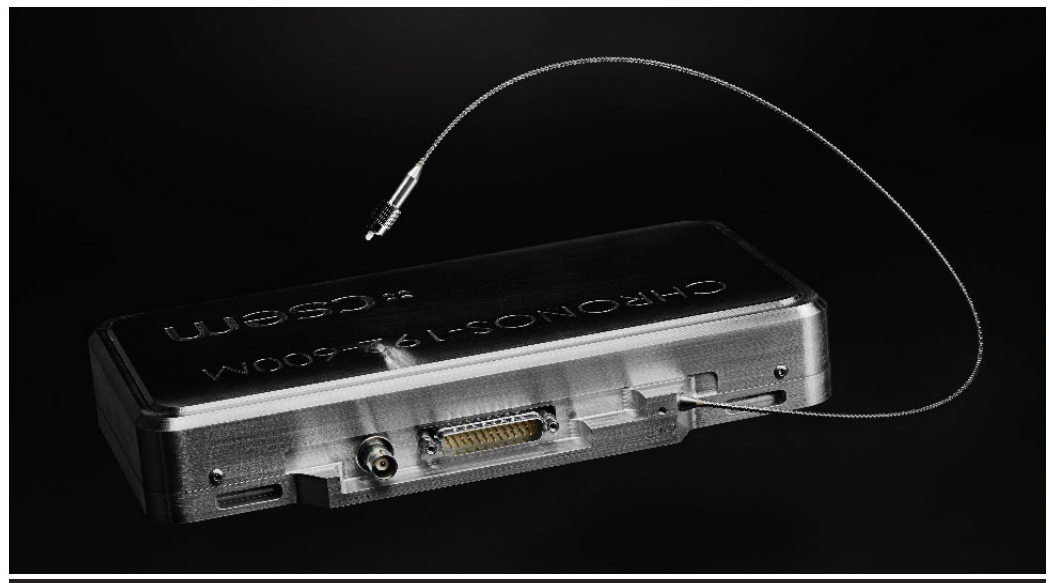

Figure 3-1: CSEM mode-locked laser head

The laser parameters are summarized in the table below:

Table 3-1 : MLL output parameters

\begin{tabular}{|l|l|l|}
\hline Parameter & Value & Comment \\
\hline Operation wavelength & $1560 \mathrm{~nm}$ & \\
\hline Repetition rate & $395.95 \mathrm{MHz}$ & Tunable repetition rate, from 395.9948 MHz to $395.8960 \mathrm{MHz}$ \\
\hline Pulse duration & $320 \mathrm{fs}$ & From optical spectrum, sech2 pulses (8 nm FWHM) \\
\hline Output power out of fiber & $20 \mathrm{~mW}$ & \\
\hline
\end{tabular}

\section{Erbium-doped fiber amplifier}

To generate the $\mathrm{f}-3 \mathrm{f}$ interferometer in the silicon nitride waveguide sufficient peak power is needed. For this reason, it was decided to implement an EDFA for amplification. The EDFA's baseline design is a so-called parabolic amplifier generating output pulses with parabolic temporal shape. Such temporal pulse shape is ideally suited for compression in a single mode fiber to obtain short pulses and thus high peak power necessary for the $\mathrm{f}_{0}$ detection. The amplifier output is split into two arms. One arm goes to the silicon nitride waveguide for $\mathrm{f}_{\mathrm{o}}$ detection and the second arm provides light for photodetection and RF generation.

The fiber length on the waveguide arm is adjusted to obtain pulses as short as possible. Pulse parameters at the waveguide input are summarized in the Table 3-2. 
Table 3-2 : Parabolic EDFA output parameters

\begin{tabular}{|l|l|l|}
\hline Parameter & Value & Comment \\
\hline Operation wavelength & $\begin{array}{l}1560 \mathrm{~nm} \\
\Delta \lambda=45 \mathrm{~nm}, \text { FWHM }\end{array}$ & \\
\hline Repetition rate & $395.95 \mathrm{MHz}$ & \\
\hline Pulse duration & $97 \mathrm{fs}$ & From autocorrelation measurement \\
\hline Output power out of fiber & $166 \mathrm{~mW}$ & \\
\hline
\end{tabular}

Considering the parameters displayed in Table 3-2, an optical peak power of more than $4 \mathrm{~kW}$ or pulse energy of more than $400 \mathrm{pJ}$ is reached at the input of the PIC waveguide which is more than sufficient to generate the required supercontinuum spectral bandwidth.

\section{Pulse interleaver}

The part used for the optical RF generation is connected to a fiber interleaver described in the following.

The laser has a repetition rate close to $395 \mathrm{MHz}$. In order to get maximum RF power into the 24th repetition rate harmonic $(9.5 \mathrm{GHz})$, the laser repetition rate is multiplied using a three-stage fiber based interleaver multiplying the repetition rate by a factor of 8 to $3.16 \mathrm{GHz}$ (see Figure 3-2). The interleaver is made of PM fiber and, in order to maximize the power for photodetection, the last combiner stage (S3 in Figure 3-2) is a polarizing beam combiner leading to alternate and orthogonal polarization of the output pulses [6]. The 3.16-GHz optical pulse train then impinges onto the HPPD, which detects the fundamental of the $3.16 \mathrm{GHz}$ repetition rate pulses, its harmonics, and the spurious harmonics of the $395-\mathrm{MHz}$ repetition rate of the laser. The third harmonic of the $3.16 \mathrm{GHz}$ is isolated by an RF bandpass filter. In addition and to generate the best phase noise purity of the $9.5 \mathrm{GHz}$ signal, sub-ps optical pulses impinging the HPPD are important to keep the phase noise reduction thanks to shot noise correlation [7]. In order to achieve this, a patch cord of dispersion compensating fiber (DCF) allows to recompress the pulses at the output of the fiber interleaver (several ps pulse duration). A measured pulse duration of $143 \mathrm{fs}$ was achieved at the output of the interleaver.

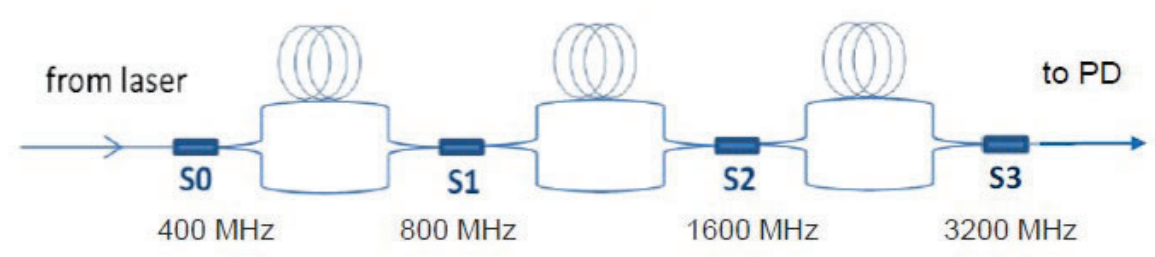

Figure 3-2 :Fiber interleaver multiplying the laser repetition rate by a factor of 8 to $3.16 \mathrm{GHz}$.

\section{Silicon nitride waveguide}

The design of the nonlinear waveguides is based on finite element numerical simulations that yield group velocity dispersion as well as nonlinear optical characteristics for specific waveguide geometries. While the height of the waveguide was fixed to $800 \mathrm{~nm}$ (corresponding to Ligentec's AN800 fabrication process), the (top) width was varied from $200 \mathrm{~nm}$ to $3000 \mathrm{~nm}$, a sidewall angle of $1.5^{\circ}$ was assumed for all geometries. Based on the simulated waveguide geometries, the super-continuum generation process was simulated using split-step simulation of the nonlinear optical dynamics in the waveguide. As a result of the simulation, 44 different waveguides have been chosen for the fabrication runs in a $5 \times 10 \mathrm{~mm}^{2}$ large chip. While the first fabrication run relied on standard fiber-to-chip coupling by means of lensed fibers in a straight 
waveguide, in a second fabrication run SMF-28 single mode fiber compatible couplers (ExSpot from Ligentec) with better alignment tolerance and power handling capability were implemented at the waveguides input and output.

A broad super continuum spanning over hundreds of nanometers is observed. It was difficult to judge from the output spectra whether an output spectrum satisfies the conditions for $\mathrm{f}_{0}$ detection. For that reason, the output of the PIC waveguides was analyzed with a photodiode. Three waveguides allowed for detection of $\mathrm{f}_{0}$.

\section{High-power handling photodiode}

The used HP-PD was developed by Albis Optoelectronics in the ESA ARTES project HOPP in collaboration with CSEM (see previously mentioned contribution to this conference). The HP-PD used in this work was optimized for $10 \mathrm{GHz}$ microwave generation. The main optimization parameter is the active diameter while the epitaxial structure was not modified with respect to the HOPP project. Indeed, a large photodiode diameter results in better heat management of the photodiode which yields better power handling capabilities and increased optical damage threshold. Furthermore, a large diameter allows to spread the optical input power across a larger area which allows high optical power operation without saturation effects taking place. On the other hand, a larger device diameter also results in a larger device capacitance which reduces the device bandwidth. The bandwidth must of course not be reduced below the targeted carrier frequency. The design rule derived from this consideration is that the PD bandwidth should be a factor of 1.4-1.5 above the targeted RF frequency - in our case about $14 \mathrm{GHz}$. The device responsivity is around $0.5 \mathrm{~A} / \mathrm{W}$. The HP-PD is mounted in a hermetic package with an optical fiber feedthrough on one side and a coaxial K-connector on the other side. The electrical feedthrough (RF output) is terminated by an internal matching impedance of $50 \Omega$. The bias voltage is applied to the HPPD via two pins on the side of the package through an integrated bias-T. The HP-PD is aligned to a single mode optical fiber through the optical feedthrough to get the maximum responsivity.

\section{COMPLETE SYSTEM}

The system was assembled and tested in the CSEM laboratory. Pictures of the setup are shown in Figure 4-1. 


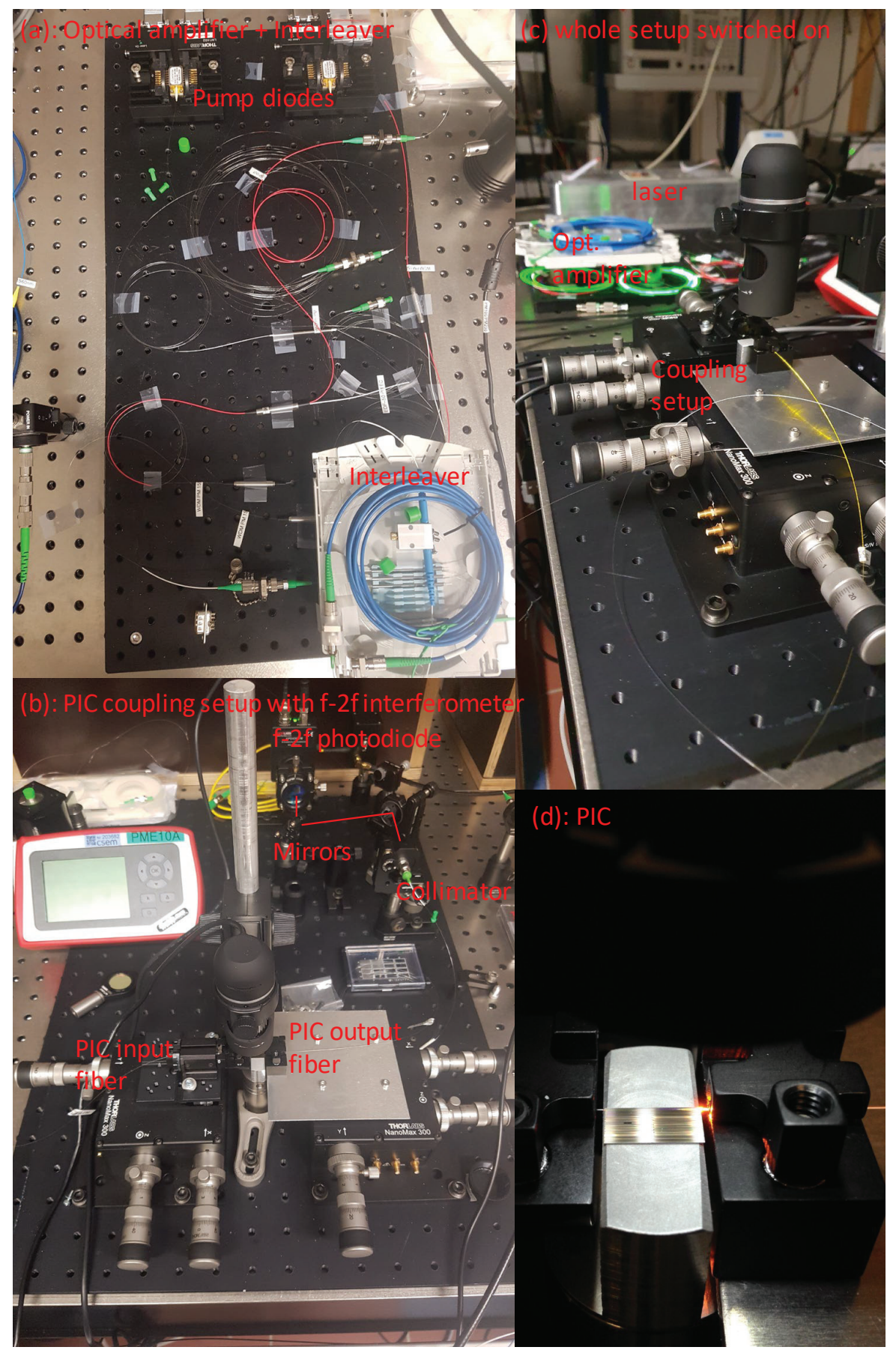

Figure 4-1 :Pictures of the photonics oscillator setup: (a) Optical amplifier + interleaver, (b) PIC coupling setup with $f-2 f$ interferometer,(c) whole setup and (d) zoom onto the PIC in action 


\section{SYSTEM TESTING AND PERFORMANCE}

\section{$\mathbf{f}_{\text {rep }}$ and $\mathbf{f}_{0}$ stabilization}

The part of the laser light used for the stabilization of $\mathrm{f}_{\text {rep }}$ was optically filtered by a narrow ( $\left.1 \mathrm{~nm}\right)$ optical band-pass filter and combined with the light coming from an ultra-stable CW laser which was itself locked onto a high-finesse Fabry-Pérot cavity (Finesse 200'000 and $10 \mathrm{~cm}$ length with fused-silica mirror substrates and ULE compensation rings) with the PoundDrever-Hall technique. The optical beat note generated by the mixing of the CW laser and one mode-locked laser comb line was detected by a photodiode and band-pass filtered. A synthesizer stabilized to an active hydrogen maser served as a local oscillator for the $f_{\text {rep }}$ control loop. A homemade analog PLL electronics acting on a piezo-electric transducer mounted in the MLL cavity was used for the $f_{\text {rep }}$ stabilization.

The RF spectrum of the beat note frequency has servo bumps at $100 \mathrm{kHz}$ away from the coherent peak. This high locking bandwidth for a piezo actuator is possible thanks to the careful mechanical design of the CSEM MLL. The phase noise $\mathrm{L}(\mathrm{f})$ measurements of the $\mathrm{f}_{\text {rep }}$ lock alone and when the $\mathrm{f}_{0}$ lock is engaged as well are shown in the left panel of Figure 5-1. The servo bump at around $100 \mathrm{kHz}$ is present as well as other spurious noise features at $\mathrm{f}<100 \mathrm{kHz}$. The integrated phase noise of the beat note frequency integrated between $1 \mathrm{~Hz}$ and $1 \mathrm{MHz}$ amounts to $224 \mathrm{mrad}$ in case of $\mathrm{f}_{\text {rep }}$ stabilization only and $220 \mathrm{mrad}$ when $\mathrm{f}_{0}$ is stabilized at the same time.

The part of the laser light used for the stabilization of $\mathrm{f}_{0}$ was coupled to a Ligentec $\mathrm{SiN}$ waveguide. The output of the waveguide was coupled to a fibre and the fibre output was focused onto a photodetector. The generated RF signal was band-pass filtered and a very similar PLL as compared to the $\mathrm{f}_{\text {rep }}$ lock was implemented. It acts on the current of the MLL pump laser.

The $\mathrm{f}_{0}$ RF spectrum exhibits servo bumps at $30 \mathrm{kHz}$ from the coherent peak. The RF spectrum is clean and does not exhibit spurs. The phase noise double-sideband spectral density $\mathrm{L}(\mathrm{f})$ of the $\mathrm{f}_{0}$ lock alone and simultaneously with the $\mathrm{f}_{\text {rep }}$ lock is shown in Figure 5-1, on the right panel. The servo bump at around $30 \mathrm{kHz}$ is present as well and the spectrum shows only a few weak spurs. As the loop gain for the $\mathrm{f}_{0}$ lock alone is slightly higher the phase noise is here about $10 \mathrm{dBc} / \mathrm{Hz}$ lower at intermediate frequencies than in the case for the two combined $f_{0}$ and $f_{\text {rep }}$ locks. The servo bump of the $f_{\text {rep }}$ lock at around $100 \mathrm{kHz}$ can also be observed in the case where the two locking loops are active. $\mathrm{f}_{0}$ integrated phase noise amounts to $912 \mathrm{mrad}$ in case of $\mathrm{f}_{0}$ stabilization only and $960 \mathrm{mrad}$ when $\mathrm{f}_{\mathrm{rep}}$ is stabilized at the same time.
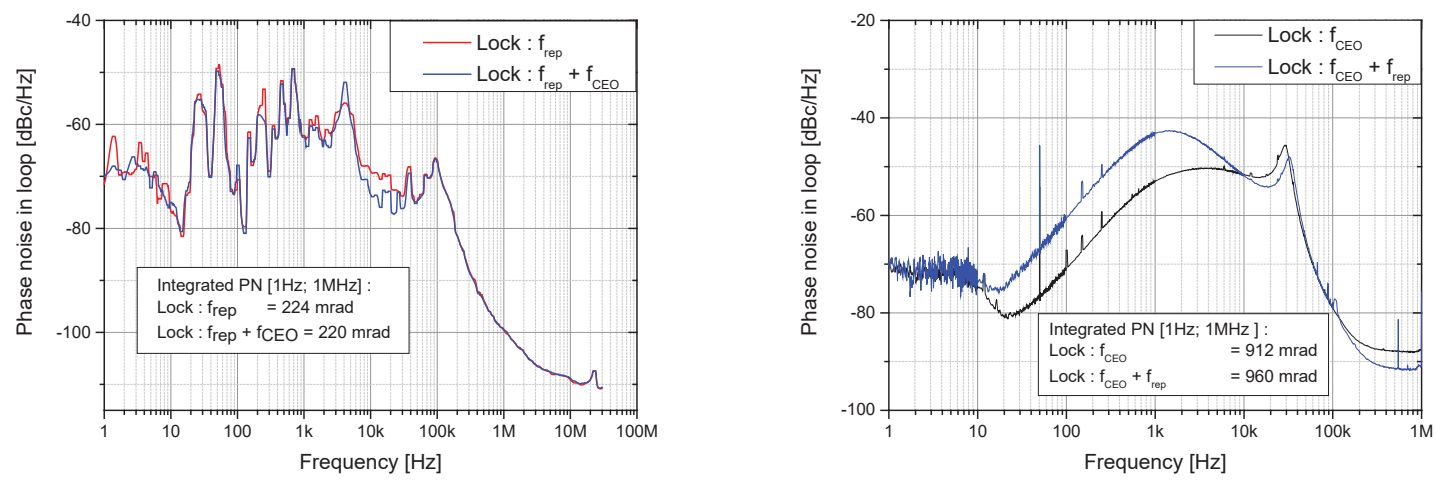

Figure 5-1: Left: in loop phase noise of frep signal; Right: in loop phase noise of fo signal.

\section{High frequency phase noise measurement}

In a first experiment, the HP-PD was illuminated with $15 \mathrm{~mW}$ of optical power. The photodiode bias voltage was varied from 8 to 15 volts and the phase noise was measured from $1 \mathrm{kHz}$ to $30 \mathrm{MHz}$ offset frequency (see Figure 5-2 below). The measurement is depicted in Figure 5-2. At low offset frequencies the phase noise is almost the same for all bias voltages. At offset frequencies above $10 \mathrm{kHz}$ it can be observed that the higher the bias voltage the lower the phase noise. For the 
lowest bias voltage, the phase noise floor amounts to $-169 \mathrm{dBc} / \mathrm{Hz}$ whereas at a bias voltage of $15 \mathrm{~V}$ noise floor is significantly improved to $-175 \mathrm{dBc} / \mathrm{Hz}$.

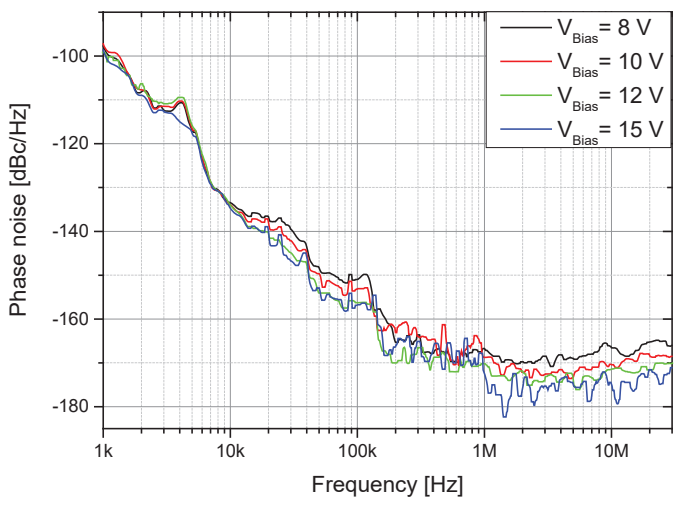

Figure 5-2 : Phase noise measurements versus different photodiode bias voltages

\section{Phase noise for different locking configurations}

In a last set of measurements, the influence of the $f_{0}$ and $f_{\text {rep }}$ stabilization on the microwave signal phase noise was tested. Those measurements were all carried out at a bias voltage of $15 \mathrm{~V}$ and a photocurrent of $8.2 \mathrm{~mA}$. The RF power in the 9.5 GHz tone was about $-0.5 \mathrm{dBm}$. The measurements are shown in Figure 5-3.

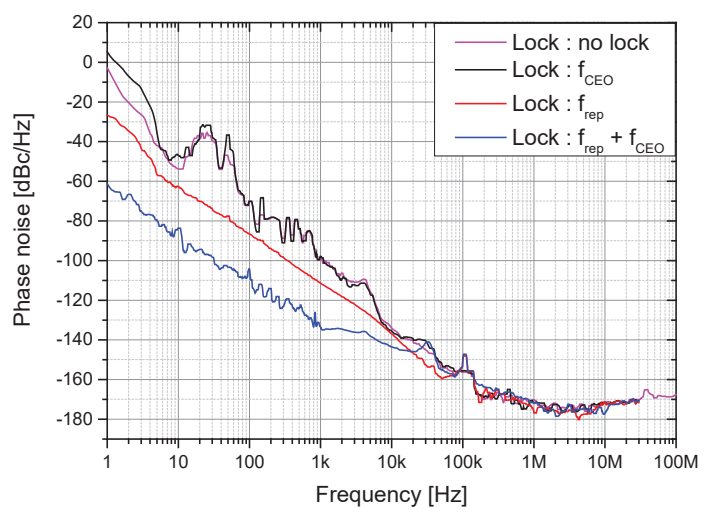

Figure 5-3 : Phase noise of $9.491 \mathrm{GHz}$ tone with and without all combinations of $f_{0}$ and $f_{\text {rep }}$ stabilization $/ P_{R F} \approx-0.5 \mathrm{dBm}$

In the case of a free running laser, the phase noise is on the order of $0 \mathrm{dBc} / \mathrm{Hz}$ at $1 \mathrm{~Hz}$ offset frequency and about $-140 \mathrm{dBc} / \mathrm{Hz}$ at $10 \mathrm{kHz}$ offset frequency. It continues to decay up to about $1 \mathrm{MHz}$ where the phase noise floor of about $-175 \mathrm{dBc} / \mathrm{Hz}$ is reached. When $\mathrm{f}_{0}$ stabilization is activated the phase noise curve is essentially the same as with the free running laser. When the optical $f_{\text {rep }}$ lock is activated without $f_{0}$ stabilization, the phase noise significantly drops up to a frequency of about $100 \mathrm{kHz}$ which corresponds to the lock bandwidth of the $\mathrm{f}_{\text {rep }}$ lock. This indicates that low offset

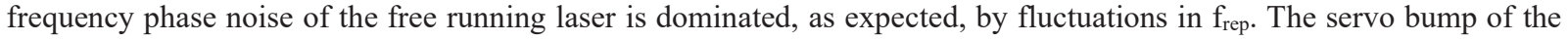

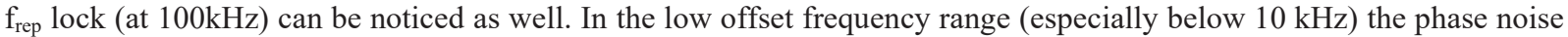
limit is attributed to acoustic noise and vibrations causing laser cavity length fluctuations. At offset frequencies above $100 \mathrm{kHz}$ the phase noise is the same as for the case without repetition rate stabilization. When the $\mathrm{f}_{0}$ stabilization is activated together with the $\mathrm{f}_{\text {rep }}$ stabilization, the phase noise further decreases by up to $30 \mathrm{~dB}$ within the lock bandwidth of 
the $f_{0}$ lock of $30 \mathrm{kHz}$. Both the servo bump of the $f_{\text {rep }}$ lock and the $f_{0}$ lock can be observed on the phase noise curve. The phase noise floor remains unaffected by the servos.

These phase noise measurements were performed with a Rohde-Schwartz FSWP using cross-correlation. While this instrument provides state-of-the-art capabilities in a commercial instrument, it is not capable to measure the ultra-low phase noise of the photonics oscillator up to frequencies of about $10 \mathrm{kHz}$ since a prohibitively high number of crosscorrelations would be needed. Other setups are required to measure such low phase noise which is expected to be in the range of $-100 \mathrm{dBc} / \mathrm{Hz}$ at $1 \mathrm{~Hz}$ offset corresponding to the $1 \mathrm{~s}$ relative instability of the reference cavity of about $10^{-15}$ [8].

\section{CONCLUSION}

A photonics-based microwave oscillator relying on a passively mode-locked diode-pumped solid-state laser and photodetection in a high-power handling photodiode has demonstrated ultra-low phase noise microwave generation at a carrier frequency of $9.5 \mathrm{GHz}$. The system incorporates several key features like a parabolic EDFA, a fully-PM pulse interleaver and a silicon nitride waveguide. The silicon nitride waveguide allows a monolithically integrated f- $3 \mathrm{f}$ nonlinear interferometer for $\mathrm{f}_{0}$ detection. The measured phase noise exhibited an ultra-low phase noise floor of $-175 \mathrm{dBc} / \mathrm{Hz},-157 \mathrm{dBc} / \mathrm{Hz}$ at $100 \mathrm{kHz}$ and $-142 \mathrm{dBc} / \mathrm{Hz}$ at $10 \mathrm{kHz}$ offset frequencies. Such an approach offers great potential for future application on-board satellites where phase noise beyond what is achievable with quartz technology is needed and where optical clock distribution is advantageous. As a key asset of the selected approach, the mode-locked laser, the photodiode and the silicon nitride waveguide provided good preliminary results regarding radiation hardness.

Research funding: This work has been supported by the Mesure de Positionnement project Spacewave of the Swiss Space Office and the Canton of Neuchâtel.

Conflict of interest: The authors declare no conflicts of interest regarding this contribution.

\section{REFERENCES}

[1] L. Maleki, «The optoelectronic oscillator,» Nature Photonics, 5, p. 728-730, 2011.

[2] M. Alouini, M. Brunel, F. Bretenaker, M. Vallet und A. Le Floch, «Dual tunable wavelength Er:Yb:glass laser for terahertz beat frequency generation,",» IEEE Photonics Technol. Lett., 10, p. 1554-1556, 1998.

[3] T. Fortier, M. Kirchner, F. Quinlan, J. Taylor, J. Bergquist, T. Rosenband, N. Lemke, A. Ludlow, Y. Jiang, C. Oates und S. Diddams, «Generation of ultrastable microwaves via optical frequency division,» Nature Photonics, 5, pp. 425-429, 2011.

[4] T. D. Shoji, W. Xie, K. L. Silverman, A. Feldman, A. Harvey, R. P. Mirin und T. R. Schibli, «Ultra-low-noise monolithic mode-locked solid-state laser,» Optica, 3, pp. 995-998, 2016.

[5] E. Portuondo-Campa, R. Paschotta und S. Lecomte, «Sub-100 attosecond timing jitter from low-noise passively mode-locked solid-state laser at telecom wavelength,» Optics Letters, 38, pp. 2650-2653, 2013.

[6] E. Portuondo-Campa, G. Buchs, S. Kundermann, L. Balet und S. Lecomte, «Ultra-low phasenoise microwave generation using a diode-pumped solid-state laser based frequency comb and a polarization-maintaining pulse interleaver,» Optics Express, 23, pp. 32441-32451, 2015.

[7] F. Quinlan, T. Fortier, H. Jiang, A. Hati, C. Nelson, Y. Fu, J. Campbell und S. Diddams, «Exploiting shot noise correlations in the photodetection of ultrashort optical pulse trains,» Nature Photonics, 7, p. 290-293, 2013.

[8] X. Xie, R. Bouchand, D. Nicolodi, M. Giunta, W. Hänsel, M. Lezius, A. Joshi, S. Datta, C. Alexandre, M. Lours, P.-A. Tremblin, G. Santarelli, R. Holzwarth und Y. Le Coq, «Photonic microwave signals with zeptosecond-level absolute timing noise,» Nature Photonics, 11, pp. 44-47, 2017. 